\title{
Recombination properties of Si-doped InGaAs/GaAs quantum dots
}

\author{
J Siegert $^{1}$, S Marcinkevičius ${ }^{1}$, L F ${ }^{2}$ and C Jagadish ${ }^{2}$ \\ ${ }^{1}$ Department of Microelectronics and Applied Physics, Royal Institute of Technology, \\ Electrum 229, 16440 Kista, Sweden \\ ${ }^{2}$ The Australian National University, Department of Electronic Materials Engineering, \\ Research School of Physical Sciences and Engineering, Canberra ACT 0200, Australia \\ E-mail: siegert@imit.kth.se
}

Received 23 August 2006, in final form 18 September 2006

Published 9 October 2006

Online at stacks.iop.org/Nano/17/5373

\begin{abstract}
The recombination properties of directly doped InGaAs/GaAs quantum dots (QDs) for application in quantum dot infrared photodetectors (QDIPs) have been investigated by time-resolved photoluminescence. Compared with undoped and barrier-doped samples, the overall effect of direct dot doping is found to be small, resulting in only slight deterioration of dot homogeneity. Low-temperature photoluminescence decay times decrease very little, indicating that direct doping does not cause a significant increase of nonradiative recombination. In addition, directly doped quantum dots show a significantly weaker quenching of the photoluminescence intensity with temperature. At the same time, barrier doping causes the formation of more and smaller dots, which results in high photoluminescence intensity at low temperatures but an early onset of thermal carrier emission from the dots. The results suggest that direct QD doping is more prospective for realizing room-temperature operation in QDIPs.
\end{abstract}

(Some figures in this article are in colour only in the electronic version)

\section{Introduction}

Devices based on self-assembled quantum dots (QDs) promise advantages over their conventional counterparts or even open the way for completely novel designs. Quantum dot applications in light emitting, detecting and electronic devices are based on particular QD properties that should be tailored for the specific purpose. Among such properties one could mention dot size and its uniformity, material composition, dot density and the number of dots and dot layers. Quantum dot doping is another adjustable parameter, which is essential for such QD devices as QD modulationdoped field-effect transistors (QD-MODFETs), QD intraband lasers [1-3] and QD infrared photodetectors (QDIPs). The latter devices are among the most popular QD applications; their key features include room-temperature operation, ability to detect light at normal incidence and excellent detectivity characteristics [4-7]. The operating principle resembles that of a photoresistor: electrons, trapped inside the QDs, can be photoexcited and contribute to the photocurrent between the electrodes of the structure. In QDIPs, accurate doping control is critical in order to fill the QDs without creating a surplus of carriers that would increase the dark current.

One commonly used method of doping is introducing a modulation-doped layer at some short distance, of the order of $10 \mathrm{~nm}$, from the QDs. A drawback of modulation doping is the problem of achieving accurate doping control since the number of carriers that are transferred from the donors into the dots is typically much less than the number of donors. Alternatively, the dots may be directly doped, which simplifies the growth procedure and allows for a better control of the number of free carriers introduced into a QD. However, direct dot doping can noticeably reduce the optical quality of QDs $[8,9]$, which manifests in decreased photoluminescence (PL) intensity and increased width of the PL peak. Since the quantum dot formation depends critically on the growth conditions, the presence of dopants in the barriers or the dots may furthermore affect the dot density, average dot size and size distribution [10-13]. While these parameters do have an influence on device operation, the critical feature 


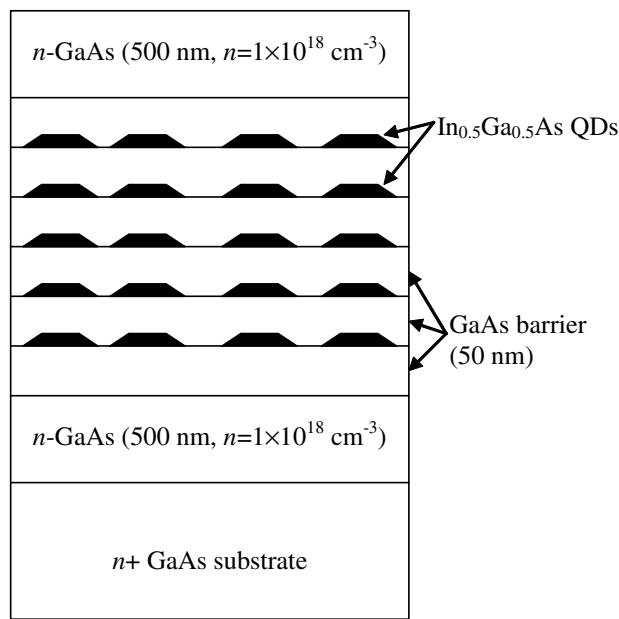

Figure 1. Schematic drawing of the sample structure (undoped sample). Additionally, the dots or the GaAs barriers separating the QD layers are doped with a nominal Si concentration $n=1 \times 10^{17} \mathrm{~cm}^{-3}$.

is nonradiative recombination that may severely degrade the performance of a QDIP. In this study, aimed at examining PL properties of directly doped InGaAs QDs, we therefore concentrate on carrier lifetimes, which are a measure of recombination processes. To get a more general picture, directly doped QDs are compared to undoped and barrierdoped QD structures.

\section{Experiments}

$\mathrm{In}_{0.5} \mathrm{Ga}_{0.5}$ As quantum dot structures were grown on $n^{+} \mathrm{GaAs}$ substrates using metal organic chemical vapour deposition (MOCVD). After deposition of $500 \mathrm{~nm} n$-doped GaAs (doping concentration $n=1 \times 10^{18} \mathrm{~cm}^{-3}$ ), 5 layers of QDs were grown, separated by $50 \mathrm{~nm}$ thick GaAs barriers. Finally, the samples were covered with a $500 \mathrm{~nm}$ thick $n$-GaAs $(n=1 \times$ $10^{18} \mathrm{~cm}^{-3}$ ) cap layer. The structure is schematically illustrated in figure 1. The QD growth parameters were identical to the ones reported in [14]. Cross-sectional transmission electron microscopy studies performed on a 15-layer QDIP grown under the same conditions shows that the dots are shaped like truncated cones [15].

In order to study the effects of doping on the QDs, three different sets of samples were prepared: (a) direct dot doping, (b) barrier doping and (c) undoped reference. In the first two sets, $\mathrm{Si}$ was introduced during the growth at a nominal concentration of $1 \times 10^{17} \mathrm{~cm}^{-3}$. Atomic force microscopy (AFM) of similar uncapped structures with only a single dot layer shows QDs with typical sizes of 20-30 nm diameter and $3-5 \mathrm{~nm}$ height. The density of the QDs is $4.4 \times 10^{10} \mathrm{~cm}^{-2}$ (undoped sample) and $6.6 \times 10^{10} \mathrm{~cm}^{-2}$ (directly dot doped samples), respectively.

Optical characterization of the structures was performed using time-resolved photoluminescence spectroscopy. A tunable mode-locked Ti:sapphire laser (pulse length $130 \mathrm{fs}$, repetition rate $76 \mathrm{MHz}$ ) was used for above $(790 \mathrm{~nm})$ and below $(890 \mathrm{~nm})$ barrier excitation. Carrier lifetimes

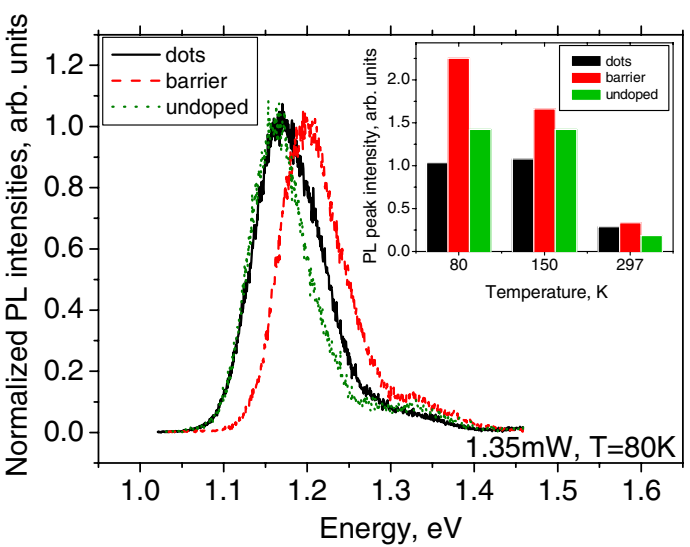

Figure 2. Normalized low-temperature time-integrated photoluminescence spectra for directly dot-doped, barrier-doped and reference samples obtained by moderate excitation into the barrier. The inset shows the PL peak intensities at different temperatures.

Table 1. Summarized results for directly dot-doped, barrier-doped and undoped reference samples. The fitted values for measurements performed at $80 \mathrm{~K}$ are $E_{\mathrm{QD}, 1}$ and $E_{\mathrm{QD}, 2}$ (the two energies associated with QD transitions when fitting the spectra with a set of three Gaussians, two for the QDs and one for the WL) and FWHM (the full width at half maximum of the QD peak). Estimated errors for the peak energies are $10 \mathrm{meV}$. The activation energy $E_{\mathrm{A}}$ was extracted from the Arrhenius plots in figure 6.

\begin{tabular}{llll}
\hline & Dots & Barrier & Undoped \\
\hline$E_{\mathrm{QD}, 1}(\mathrm{eV})$ & 1.161 & 1.191 & 1.157 \\
$E_{\mathrm{QD}, 2}(\mathrm{eV})$ & 1.215 & 1.242 & 1.214 \\
$\mathrm{FWHM}(\mathrm{meV})$ & $92 \pm 2$ & $82 \pm 2$ & $78 \pm 2$ \\
$E_{\mathrm{A}}(\mathrm{meV})$ & $148 \pm 10$ & $121 \pm 10$ & $150 \pm 10$ \\
\hline
\end{tabular}

were measured by detecting the photoluminescence signal with a synchroscan streak camera combined with a $0.25 \mathrm{~m}$ spectrometer. The experiments were carried out at various temperatures in the range from 80 to $297 \mathrm{~K}$ and at different excitation powers. The temporal resolution for the timescales used was about 40 ps.

\section{Results and discussion}

Figure 2 shows normalized time-integrated $80 \mathrm{~K}$ spectra of the three samples measured at moderate excitation power. The main peak is related to transitions within the QDs whereas the small shoulder at around $1.33 \mathrm{eV}$ is from the wetting layer (WL). These peaks can be fitted by a set of three Gaussians (two for the QD peak, one for the WL). The parameters of these spectra are given in table 1. Compared to the undoped reference sample, the spectra of the doped structures exhibit (a) a blue shift and (b) broadening of the QD peak. Several effects should be considered in order to explain the shift: band filling [8], screening of a built-in piezoelectric field [8], repulsive Coulomb interactions of holes and donors in the dots and, finally, different dot sizes [11]. Which of these offers the most reasonable explanation in our case can be qualitatively evaluated from the dependence of the PL peak width on the excitation power. For example, if the blue shift was caused by screening of a piezoelectric field, the peak width would 

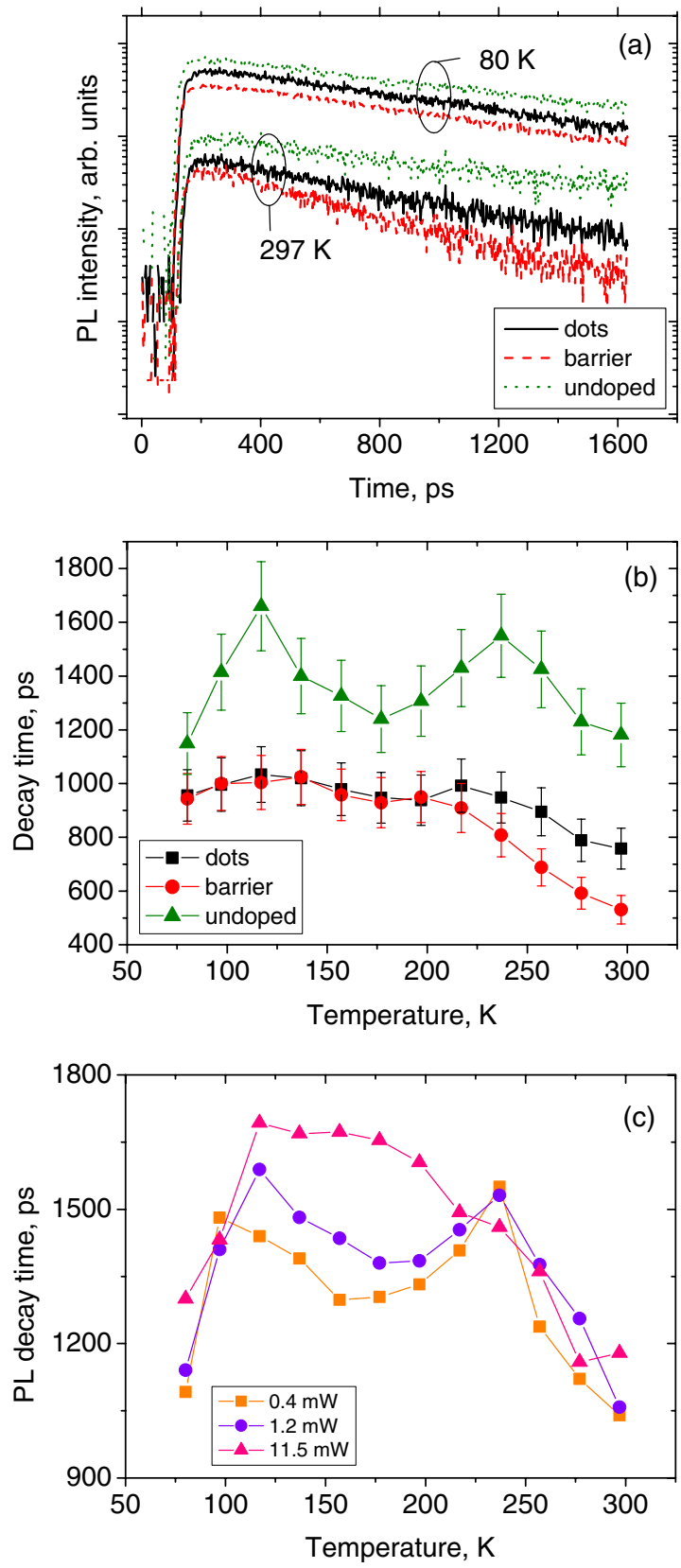

Figure 3. (a) Photoluminescence transients at 80 and $297 \mathrm{~K}$ (b) Dependence of the photoluminescence decay time (extracted from single exponential fits to the PL transients) on temperature. The samples were excited above the GaAs bandgap with an average power of $0.93 \mathrm{~mW}$. (c) Dependence of the PL decay time on temperature for the undoped sample at a few selected excitation powers. For viewing convenience, the error bars (estimated errors are $10 \%$ of the PL decay time) are not displayed in (c).

decrease with increased photoexcitation, whereas band filling effects manifest themselves in PL peak broadening [16]. One would, therefore, expect different trends of the peak width as a function of excitation power between doped and undoped samples, which is not observed in our experiment. On the other hand, Si doping in the barrier layer or throughout the InGaAs QD layer may cause a decrease of the average dot size [13] and larger size inhomogeneity $[9,12]$. This explanation fits our

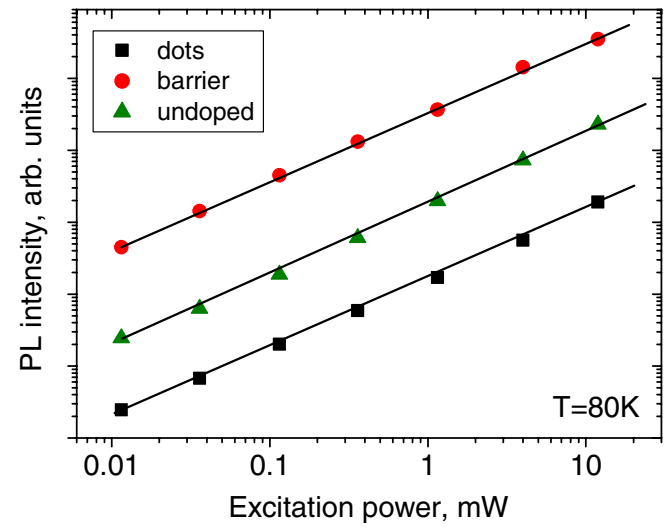

Figure 4. Time-integrated photoluminescence peak intensities measured at different above-barrier excitation conditions and $T=80 \mathrm{~K}$. For clarity, the plots are shifted in the vertical direction relative to each other. The slope of the lines is $0.96 \pm 0.05$, $0.97 \pm 0.05$ and $1.00 \pm 0.05$ for dot-doped, barrier-doped and undoped samples, respectively.

experimental results; hence, we attribute the peak blue shift and broadening in the doped samples to variations of dot geometry and strain environment rather than carrier filling effects.

The inset of figure 2 shows the time-integrated peak intensities of all our samples at a few selected temperatures. It is quite remarkable that the PL intensity (determined by the concentration of minority carriers, i.e., holes in our case) at low temperatures is considerably higher for the barrier-doped sample. Here again, there are several possible mechanisms that could be responsible for this effect. For example, barrier band bending and resulting built-in electric field could result in a better hole collection into the dots. However, additional experiments performed with below-barrier excitation reveal essentially the same relative intensities between the samples. We can therefore rule out differences in the hole capture efficiency. Nonradiative processes cannot account for the variation in low-temperature PL intensities either, since the barrier-doped sample exhibits the strongest PL intensity but shortest decay time (see figures 3 and 4). Hence, the most probable cause for an increased PL intensity is a larger number of dots. Indeed, moderate doping into the barriers has been observed to increase the QD density [13].

Photoluminescence transients obtained at $\approx 1 \mathrm{~mW}$ excitation power can be seen in figure 3(a). Decay times extracted from single-exponential fits of the transients at different temperatures are gathered in figure 3(b). The peculiar double-peak shape in figure 3(b) can, in fact, be interpreted as a single peak with a dip in the medium temperature range. This dip in the PL intensity can be associated to activation and subsequent deactivation of a carrier trap. Numerous traps have been reported in $\operatorname{In}(\mathrm{Ga}) \mathrm{As} / \mathrm{GaAs}$ quantum well and quantum dot interfaces, some of which are nearly resonant with the QD levels [17]. Then, thermal increase of the trapping rate can be caused by trap ionization. The subsequent decrease in the trapping rate may be determined by temperaturedependent decrease in the capture cross section, tunnelling rate, etc [18]. This interpretation is supported by the measurements performed at different excitation powers (figure 3(c)): with increased power, the dip disappears, obviously because of trap 


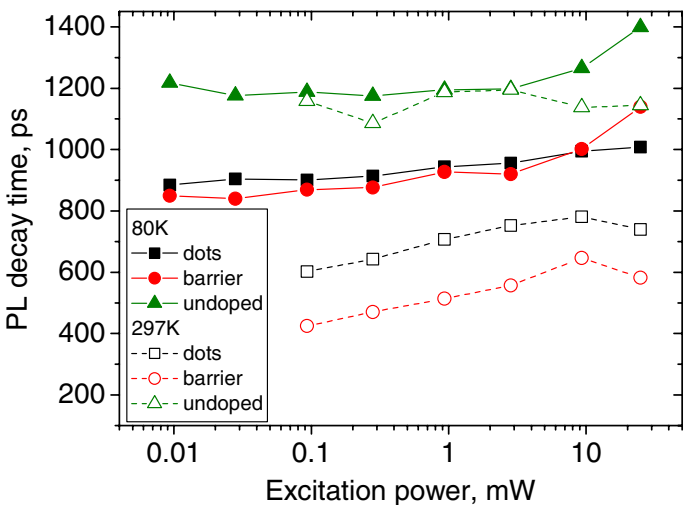

Figure 5. Photoluminescence decay times as a function of excitation power at 80 and $297 \mathrm{~K}$.

saturation. In the doped structures, this dip is much less prominent. This can be understood by taking into account a competing influence of another, more efficient, recombination channel that causes the overall decrease of the PL decay time (figure 3(b)).

At low temperatures, nonradiative recombination is not very prominent, which is indicated by fairly long decay times for all samples. This is also confirmed by the linear dependence of PL intensity on excitation power (see figure 4). It has been shown that this is the case for structures in which radiative recombination prevails, whereas in the case of dominating nonradiative processes the relationship is $I_{\mathrm{PL}} \propto I_{\mathrm{ex}}^{2}[19]$.

Still, there is a noticeable decrease in the carrier lifetimes of the doped structures compared to the undoped one, which should be attributed to nonradiative recombination at defects introduced during the QD growth. It has been shown that doping in III-V semiconductors results in a change of the nature and concentration of point defects [20]. For example, the concentration of group III vacancies $\left(V_{\mathrm{III}}\right)$ is largely increased in $n$-doped structures as a result of the Fermi level being moved towards the electron levels. It is therefore very likely that these and other point defects, which act as nonradiative recombination centres [21-23], are the cause for the observed decreased decay times in the doped QD structures.

Although no quantitative measure of the defect origin or density can be given, the sharp increase of decay time with increased excitation power is an indication that the nonradiative recombination channel can be saturated in the barrier-doped sample at low temperatures and high excitation (figure 5). The directly doped dots, on the other hand, exhibit no such feature and the decay times become longer as the excitation is increased. Evidently, a somewhat larger concentration of defects prohibits saturation of the nonradiative recombination process. Additional nonradiative channels become available at high temperatures (figure 5) and saturation with excitation is no longer observed in any of the samples.

The doped structures exhibit identical low-temperature decay times. However, at temperatures $>200 \mathrm{~K}$ striking differences between the decay times become apparent: clearly, nonradiative recombination is more readily thermally activated in the barrier-doped sample. The inset in figure 2 is a further evidence for this observation since it shows the strongest

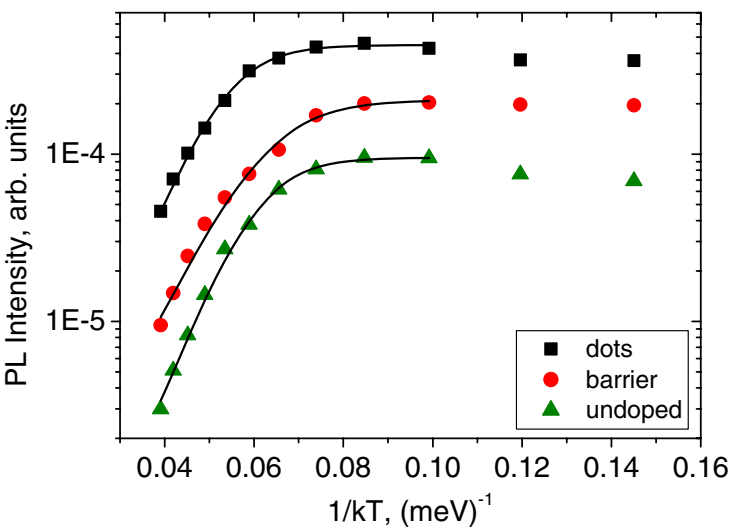

Figure 6. Temperature dependence of the integrated photoluminescence intensity. Above-barrier excitation was used at a power of $0.4 \mathrm{~mW}$. Lines are fits from which activation energies were extracted. The curves are shifted for clarity.

decrease of the PL intensity for the sample doped in the barriers. Measurements of the PL intensity as a function of temperature allow evaluation of the activation energy. We use a simplified model that considers a two level system [24]. Level 1 is the radiating exciton ground state in the QD while level 2 acts as an exciton/electron-hole pair sink. The occupation of these levels is governed by the Boltzmann law, giving the following expression for the PL intensity dependence on temperature:

$$
I(T)=\frac{I_{0}}{1+C \exp \left(-E_{\mathrm{A}} / k T\right)} .
$$

Here $I_{0}$ is the PL intensity at $T=0 \mathrm{~K}, C$ is a temperatureindependent constant, $E_{\mathrm{A}}$ is the activation energy and $k$ is the Boltzmann constant. From the fits of the data shown in figure 6 we extract activation energies of 148,121 and $150 \mathrm{meV}$ for the dot-doped, barrier-doped and undoped samples, respectively. These energies suggest thermal carrier escape to the WL and subsequent nonradiative recombination there [25].

\section{Conclusions}

In conclusion, we have studied the influence of positionselective Si doping of $\mathrm{In}_{0.5} \mathrm{Ga}_{0.5}$ As QD structures on the spectral and dynamical properties of QD photoluminescence. Barrier doping leads to an increased dot density while the average QD size is decreased. This results in a lesser carrier confinement and more efficient thermal emission. Nonradiative recombination channels, introduced as a result of doping, are saturated at high excitation at low temperatures. While the low-temperature PL intensity is the strongest in these structures due to the increased dot density, it decreases considerably with temperature.

Direct doping, on the other hand, causes a slight deterioration of the QD homogeneity, evidenced by an increased PL peak width. While there are signs that nonradiative recombination is more pronounced than in the undoped sample, the overall influence of the nonradiative recombination on the photoluminescence properties is minor. Only a small decrease of the decay times is found and, most 
remarkably, quenching of the PL intensity with temperature is much weaker than in any of the other samples. This makes direct QD doping prospective for QDIPs operating at room temperature. From a commercial point of view, this would be a major technological breakthrough because so far roomtemperature operation could only be demonstrated for devices grown by MBE [26, 27].

\section{Acknowledgment}

L Fu and C Jagadish would like to acknowledge the financial support from the Australian Research Council.

\section{References}

[1] Dmitriev I A and Suris R A 2005 Phys. Status Solidi a 202987

[2] Vukmirović N, Ikonić Z, Jovanović V D, Indjin D and Harrison P 2005 IEEE J. Quantum Electron. 411361

[3] Sauvage S and Boucaud P 2006 Appl. Phys. Lett. 88063106

[4] Rogalski A 2003 Prog. Quantum Electron. 2759

[5] Chakrabarti S, Stiff-Roberts A D, Bhattacharya P and Kennerly S W 2004 J. Vac. Sci. Technol. B 221499

[6] Ryzhii V, Khmyrova I, Ryzhii M and Mitin V 2004 Semicond. Sci. Technol. 198

[7] Fu L, Lever P, Sears K, Tan H H and Jagadish C 2005 IEEE Electron. Device Lett. 26628

[8] Phillips J, Kamath K, Zhou X, Chervela N and Bhattacharya P 1998 J. Vac. Sci. Technol. B 161343

[9] Wang H L, Yang F H and Feng S L 2000 J. Cryst. Growth 21235

[10] Saucedo-Zeni N, Zamora-Peredo L, Gorbatchev A Yu, Lastras-Martínez A, Balderas-Navarro R, Medel-Ruiz C I and Méndez-García V H 2003 J. Cryst. Growth 251201
[11] Nah J 2005 J. Vac. Sci. Technol. 231047

[12] Qian Z, Songlin F, Dong N, Haijun Z, Zhiming W and Yuanming D 1999 J. Cryst. Growth 200603

[13] Park Y M, Park Y J, Kim K M, Lee J I and Yoo K H 2005 Physica E 25647

[14] Fu L, Lever P, Sears K, Tan H H and Jagadish C 2005 IEEE Electron. Device Lett. 26628

[15] Fu L, Tan H H, McKerracher I, Wong-Leung J, Jagadish C, Vukmirović N and Harrison P 2006 J. Appl. Phys. 99114517

[16] Ma W, Wang X, Wang Z, Hussein M L, Schultz J, Xiao M and Salamo G J 2003 Phys. Rev. B 67035315

[17] Wang J S, Chen J F, Huang J L, Wang P Y and Guo X J 2000 Appl. Phys. Lett. 773027

[18] Abakumov N, Perel V I and Yassievich I N 1994 Nonradiative Recombination in Semiconductors (Amsterdam: North-Holland)

[19] Marcinkevičius S, Olin U and Treideris G 1993 J. Appl. Phys. 743587

[20] Shchekin O B, Deppe D G and Lu D 2001 Appl. Phys. Lett. 783115

[21] Walther C, Bollmann J, Kissel H, Kirmse H, Neumann W and Masselink W T 1999 Physica B 273/274 971

[22] Sobolev M M, Kochnev I V, Lantratov V M, Cherkashin N A and Emtsev V V 1999 Physica B 273/274959

[23] Siegert J, Gaarder A, Marcinkevičius S, Leon R, Chaparro S, Johnson S R, Sadofyev Y and Zhang Y H 2003 Physica E 18541

[24] Bimberg D and Sondergeld M 1971 Phys. Rev. B 43451

[25] Gurioli M, Vinattieri A, Zamfirescu M, Colocci M, Sanguinetti S and Nötzel R 2006 Phys. Rev. B 73085302

[26] Su X, Chakrabarti S, Bhattacharya P, Ariyawansa G and Perera A G U 2005 IEEE J. Quantum Electron. 41974

[27] Bhattacharya P, Su X H, Chakrabarti S, Ariyawansa G and Perera A G U 2005 Appl. Phys. Lett. 86191106 